\title{
Christian Calliess Perspektiven für eine Weiterentwicklung der Europäischen Union zu einer ökologischen Rechtsgemeinschaft*:
}

\section{Einführung}

Die Industriegesellschaft hat sich zur Risikogesellschaft gewandelt. Dieser von U1rich Beck geprägte Begriff bezcichnct eine Gesellschaft, deren Institutionen vor der Gefahr ciner drohenden Vernichtung von Umwelt bzw. Mitwelt und Mensch durch die schleichende Umweltkatastrophe' versagen. Sie versagen nach der Ansicht Becks, weil die für die Industriegesellschaft entwickeltcn Rechts- und Handlungskategorien dic Realität der Risikogcscllschaft verfehlen. Diese "Vcrwechselung der Jahrhunderte "habe »die organisierte Unverantwortlichkeit " zur Folge, ein System, in $\mathrm{dem}$ "man etwas tun und wcitertun kann, ohne es persönlich verantworten zu müssen «, ein System, in dem sich die Ursachen in einer allgemeinen Wechselhaftigkeit von Akteuren und Bedingungen, Reaktionen und Gegenreaktionen verlören. Allcs Handeln auf dem Boden des geltenden Rechts habe demnach nur die Funktion, symbolisch zu beruhigen. Das Recht werde zu einer Sicherheitsfassade, die angesichts der realen Gefahren ständig vom Zusammenbruch bedroht sei. ${ }^{2}$

Vor diesem Hintergrund stellt sich die Frage, wie unser Rechtssystem auf die Risiken der Risikogesellschaft reagieren kann. Zu unserem Rechtssystem gehört auch das Reche der EG, das als vorrangiges supranationales Recht ${ }^{3}$ das nationale Recht bereits jetzt schon maßgeblich beeinflußt und vor allem zukünftig noch stärker beeinflussen wird. Ob und wie die schrittweise Herausbildung eines ökologischen Rechts auch auf EG-Ebene erfolgen könnte und müßte, wird im folgenden zu untersuchen sein.

Die EG ist kein Staat (Bundesstaat) im herkömmlichen, völkerrechtlichen Sinne. Vielmehr ist die EG in eincm ständigen Entwicklungsprozeß der Vergemeinschaftung begriffen, der in Qualitätsveränderungen in Richtung auf eine Art Bundesstaat zum Ausdruck komme. ${ }^{4}$ Mit dem Vertrag über die Europäische Union (EUV) von Maastricht hat die EG einen weiteren Schritt auf dem Weg zum europäischen Bundesstaat getan, indem dic Europäische Union (EU) vom programmatischen Ziel des Integrationsprozesses nunmehr zur Wirklichkeit geworden ist (Art. A bis F EUV).

* Der Aufsatz basiert un scinen Grundzuggen auf cinem Vortrag, den der Vertasser auf der umweltrectitlichen Fachtagung *Wege zum okologischen Rechtsstaat a des Instituts fur Uimwelurecht Bremen am 6./7. Februar 1993 in Bremen gehalten hat.

1 Vgl. z. B. Club of Rome-Bericht 1991 . Die globale Revolution, Spiegel Spezıal Nr. 2/1991, S. ${ }_{4}$ ff.; Kommission der EG, Der Zustand der Umwelt in der EG, KOM (92) endg. VOL. III vom 3. 4. I 992.

2 Risikogesellschaft. Aut dem Weg in eine andere Moderne, 1986, S. 35 ff. (43); Gegengifte. Die organisierte Unverantwortlichkeit, 1988, S. 26, 29 ff., i1 2; sowse in: Aus Politik und Zeitgeschichte B 36/89, S. 3 (sfl.).

3 Vgl. die grundlegenden lintscheidungen des EuGH Rs. 6/64 Costa/ENEL, Slg. 1964, S. 1251 und des BVerfGE 37, S. 27 ! (Solange I) und E 73, S. 339 (Solange II) sowie zuletzt allerdings das nManstrichtUrteil $\propto$ des BVerfG, kuGRZ 1993, S. 429 (438), kritisch da7u Tomuschat, EuGRZ 1993, S. 489 (494)

4 Hierzu Ipsen, EG-Recht, Tubingen 1972, S. 66 If., 187 ff.; Ress, Jus 1992, S. 98 5.; vgl. zur F.G. nach dem Unionsvertrag von Maastricht auch Pernice, DV 1993, S. 449. 
Die EU ist das Dach für die EG und die ergänzenden Politiken und Formen der

Zusammenarbeit's. Durch Art. 8 I des zum Vertrag über die Europäische Gemeinschaft (EGV) gewandelten EWGV wird eine Unionsbürgerschaft eingeführt, die einen wesentlichen Schritt zur Verdichtung des Integrationsprozesses in Richtung auf eine staatsähnliche Struktur der EU bedeutet, gerade wenn man die Bedeutung, die dem Staatsvolk für die Konstituierung eines Staates im Völkerrecht zukommt, bedenkt. ${ }^{6}$ Zieht man ferner die Bestimmungen des EUV über die Wirtschafts- und Währungsunion und die Gemeinsame Außen- und Sicherheitspolitik in Betracht, so läßt sich von "cincm point of no return « im Integrationsprozeß sprechen, der bci konsequenter Fortentwicklung in absehbarer Zeit in einen Europäischen Bundesstaat münden wird.?

\section{Rechtliche Grundlagen und Rabmenbedingungen der gegenwärtigen EG-Umweltpolitik}

\section{Rechtliche Grundlagen der gegenwärtigen EG-Umweltpolitik}

Im Laufe der siebziger Jahre entwickelte die EG "flickenteppichartig « erste Ansätzc einer Umweltpolitik, deren Rechtsgrundlage im wesentlichen auf den Art. 100 und 235 EWGV basierte. ${ }^{8}$

Mit der Einheitlichen Europäischen Akte (EEA) vom Februar 1986 und deren Titel "Umwelt« (Art. I zor-t EWGV) wurde die gemeinschaftliche Umweltpolitik nicht nur ausdrücklich und selbständig im Vertrag selbst geregelt, sondern sie wurde auch erheblich aufgewertet. Rechtspolitisch kommt dies in der vom Europäischen Rat auf seinem Treffen in Dublin (Juni 1990) formulierten Erklärung zur Umweltpolitik der EG - „Environmental Imperative« genannt - zum Ausdruck. In zwar juristisch unverbindlicher Weise wird hicrin mit deutlichen Worten zu Fortschritten bei der Lösung der drängendsten Umweltprobleme aufgefordert und mit der Bcmerkung geschlossen: „Der Mensch ist der Treuhänder der Natur, und er ist verpflichtet, dicses Erbe zum Nutzen dicser wie auch künftiger Generationen auf vernünftige Weisc zu verwalten. ${ }^{9}$

Angesichts krasser Umsctzungs- und Vollzugsdefizite auf allen Ebenen ${ }^{10}$ versucht die EG im Rahmen ihres Fünften Umweltaktionsprogramms mit dem Titcl $»$ Für eine dauerhafte und umweltgerechte Entwicklung " " die Ziele der Dubliner Erklärung in die Praxis umzusctzen. Das neuc Umweltaktionsprogramm soll von 1993 an bis zum Ende des Jahrtausends im Umweltbereich den Rahmen für die Tätigkeit der EG abstecken und darüber hinaus alle Verantwortlichen in der Gemeinschaft einbinden. Es geht insofern über einen Aufgabenkatalog für die Institutionen der EG hinaus, als es einen Schwerpunkt auf das Prinzip der gemeinsamen Verantwortung in Umwelt-

\footnotetext{
5 Ausführlich Kess, Jus 1992, S. 98 s (986 Il.).

6 Ress, (Fn. 5), S. 987.

7 Ebenso Ress, (Fn. 5), S. 991 , der von 20 bis zo Jahren ausgeht.

8 Hierzu ausfuhrlich Ress, in: Kimminich/von Lersner/Storm, Handbuch des Umwelereches, 1986, Spalte $448 \mathrm{ff}$.

9 Abgedruckt in EA 1990, S. D 396 (407 ff.).

ro Vgl. hierzu Kommission der EG, „Der Zustand der Umwete in der EG ${ }_{\kappa}$, Dok. KOM (92) 23 endg. vom 3. 4. 1992 Teil III; Roscheisen, in: Calliess/Wegener, Europasschcs Umwclercht als Chance, 1992, S. 69 f:; Lindemann/Delfs, ZUR 1993, S. 256 ff.; Ress, Vortráge im Europa-Institut der Universitat des Saarlandes, Nr. 291, S. rz 6 .

I I Dok. KOM (92) 23 endg. vom 3.4. 1992, Teil II; dazu Wagenbaur, EuZIV 1993, S. 24 I.
} 
fragen legt: Alle Akteure, von der EG angefangen, über die Regierungen der Mitgliedstaaten, die Bundesländer und Regionen, die Gemeinden, Industrie und Handel, die privaten Organisationen und Verbände bis hin zu den Bürgern, sollen in verantwortlicher Weise in umweltpolitische Maßnahmen einbezogen und eingebunden werden.

Verschiedene Bereiche werden in dem Aktionsprogramm mit besondercr Aufmerksamkcit behandelt, weil sie einen besonders starken Einfluß auf den Versuch der Verwirklichung einer dauerhaften und umweltgerechten Entwicklung haben. Der Bereich 》Industric" ist etwa von der Überwindung der Vorstellung geprägt, daß zwischen Umweltschutz und Wettbewerbsfähigkeit der Industrie ein unüberwindlicher Interessengegensatz bestehe. Zukünftig sollen neben strengen Produkt- und Produktionsnormen auch indirekte Anreize - das Programm enthält ein eigenes Kapitel über "Neue Instrumente" - zu einer umweltgerechten Entwicklung der Industrie beitragen. Dem Bereich »Energic « wird cine Schlüsselrolle für die Umweltpolitik zugewiesen: Es sollen nicht nur eine rationelle Nutzung der Energie und über Technologieprogramme der Einsatz regenerativer Energien gefördert werden, sondern zusätzlich die tatsächlichen Kosten für den Energieverbrauch an den Endverbraucher über eine $\mathrm{CO}_{2}$-Energiesteuer weitergegeben werden. Als weitere Bereiche werden im Aktionsprogramm die Verkehrs-, Agrar-, Luftreinhaltc- und Abfallpolitik sowie der Tourismus genannt. Ein weiteres Kapitel ist den Maßnahmen der Gemeinschaft im Hinblick auf weltweite Umweltprobleme gewidmet. ${ }^{12}$

Allerdings entfaltet das Umwcltaktionsprogramm - wie schon seine Bezcichnung nahelegt - keinerlei Bindungswirkung für den Gemeinschaftsgesctzgcber. Es bildet weniger einen strikt cinzuhaltenden Arbeitsplan als eine Leitlinie für dessen Tätigkeit. ${ }^{13}$ Entscheidend für die europäische Umweltpolitik wird damit die Verbindlichkeit des gemeinschaftlichen Normengefüges.

Als Zicle der gemeinschaftlichen Umweltpolitik beschrieb Art. I 3 or I EWGV in Anlehnung an die ersten Aktionsprogramme der EG die Erhaltung und den Schutz der Umwelt sowie die Verbesserung ihrer Qualität, den Schutz der menschlichen Gesundheit und die Gewährleistung einer rationcllen Verwendung der natürlichen Ressourcen. Im Hinblick auf die Rio-Konferenz (UNCED), das Montrealer Protokoll zum Schutze der Ozonschicht und allgemein die Notwendigkeit eines globalen Umweltschutzes und internationalen Umweltrechts erscheint es konsequent, wenn der ncuc Art. r 30 I I EGV die EG ergänzend auf das Ziel der "Förderung von Maßnahmen auf internationaler Ebene zur Bewältigung regionaler und globaler Umweltproblcme« verpflichtet. Dies bedeutct aber noch lange keine eigene Kompetenz dcr EG. Vielmehr scheinen die Mitgliedstaaten, wie schon im Vorfeld der Rio-Konferenz, auch in den Folgekonferenzen eifersüchtig ihre außenpolitische Kompetenz zu verteidigen. Dies ist angesichts der juristischen Gemengelage auch kein weiter schwieriges Unterfangen für die Mitgliedstaaten. ${ }^{14}$

Ebenfalls in Anlehnung an frühere Aktionsprogramme schrcibt Art. 130 r II S. 2 EGV (vormals Art. 1 zor Il S. i EWGV) einige umweltpolitische Handlungsgrundsätze wie das Vorbeugungsprinzip, das Prinzip vorrangiger Korrektur von Umweltbeeinträchtigungen an der Quelle (Ursprungsprinzip) und das Vcrursacherprinzip fest. Infolge ihrer Aufnahme in den Vertragstext sind alle drei Prinzipien zwar als bindende Rechtsprinzipien anzusehen, jedoch haben sie einen so offenen Inhalt, daß ein Rechtsverstoß kaum nachweisbar sein dürfte.

$12 \mathrm{Vgl}$. zum ganzen Wigenbaur, (Fn. I1).

13 Vgl. Kramer, in: Calliess/Wegener, Europatsches Umwclerecht als Chance, 1992, S. 33 (35); kritisch hicrzu ders.; ZUR Nr. 4 1994.

14 Ausfuhrlich hicrzu Pernice EuR 1991, S. 273. 
Grundlegend für die Verwirklichung der umweltpolitischen Ziele der EG ist die sogenannte "Querschnittsklauscl « des Art. I zor II Satz 2 EWGV, wonach die "Erfordernisse des Umweltschutzes... Bestandteil der anderen Politiken der Gemeinschaft « sind. Durch sie wird die EG in vollem Umfang zu einer Umweltgemeinschaft. Die Qucrschnittsklausel erhebt die Umweltverträglichkeit zu einem allgemcinen Rechtsgebot, so daß sogar die Auffassung vertreten wird, der Umweltschutz habe im Konfliktfall grundsätzlich Vorrang vor anderen Gemeinschaftszielen."

Art. 1 zor Abs. 2 S. 2 EWGV will zumindest verhindern, daß die Ausgestaltung eines Politikbereiches insgesamt im Widerspruch zu den Erfordernissen des Umweltschutzes steht. Die Norm verbietet damit die Verursachung erheblicher, spürbarer Umweltbeeinträchtigungen durch die weitgehend cinseitige Ausgestaltung eines Politikbcreichs. ${ }^{16}$

Nach der Verschärfung der Qucrschnittsklausel durch den EUV heißt es in dem neuen Art. I 3 or II S. 3 EGV jetzt, daß die Erfordernisse des Umweltschutzes bei der Festlegung und Durchführung anderer Gemeinschafıspolitiken einbezogen werden müssen. Die Neuformulierung, insbesondcre mit dem Wort "müssen «, könnte eine justiziable Rechispflicht zur Integration von Umweltbelangen in die anderen Politiken der EG begründen. ${ }^{77}$

Art. ı zor II S. 3 EGV macht überdics deutlich, daß umweltschützendc Regelungen auch in anderen Politikbereichen gemäß den dort vorgesehenen Verfahren in Betracht kommen. In diesem Zusammenhang ist insbesondere die Abgrenzung zwischen Maßnahmen der allgemeinen Umweltpolitik nach Art. 1 30 EWGV und binnenmarktbezogenen Maßnahmen umstritten. ${ }^{18}$ Starken Einfluß auf das Umweltrecht hat, wie schon bisher, die Rechtsangleichung, die für den Binnenmarkt in Art. rooa EGV geregelt ist. In Art. I ooa III EGV findet sich die Formulierung, nach der die EG-Kommission bei ihren Vorschlägen u. a. im Umweltschutz auf ein »hohes Schutzniveau« verpflichtet ist. Es geht also um einen Binnenmarke mit hohem Umweltschutzniveau. ${ }^{19}$ In der Literatur wird ganz überwiegend darauf abgestellt, ob der Binnenmarkt oder der Umweltschutzaspekt der angestrebten Maßnahme überwiegt, mit der Folgc, daß Art. rooa oder aber Art. r 30 S EWGV zum Tragen kommt. ${ }^{20}$ Demgegenüber sieht der Europäische Gerichtshof (EuGH) in seincr umstrittenen Titandioxid-Entscheidung ${ }^{21}$ von Art. Iooa EWGV auch solche Richtlinien crfaßt, bei denen sich Aspekte des Binnenmarktes und des Umweltschutzes mischen. Auf der Grundlage eincs weiten Binnenmarktbegriffs wird Art. Iooa EWGV in diesem Urteil mehr oder minder für allgemein anwendbar erklärt. Andernfalls würden dic Rechte des Europäischen Parlaments (EP) im Verfahren der Zusammenarbeit ihres Wesenskerns beraubt.

Folgt man der Ansicht des EuGH, dessen Argumentation auch nach dem Vertrag von Maastricht mit Blick auf das in Art. $100 a$ EGV neu eingeführte, die Rechte des EP verstärkende Mitentscheidungsverfahren gültig bleibt ${ }^{22}$, so verbleiben für den

is Str., so Scheuing, EuR 1989 , S. 152 (177).

16 Scheuing, in: Behrens/Koch, Umweltschutz in der EG, 1991, S. 57; ausfuhrlich Jahns-Bohm/Breier, EuZW 1992, S. s rff.; Calliess, IUR 1992, S. 219 (223 f.).

I7 Dazu Calliess, IUR 1992, S. 219 (223 f.); in dieser Richtung wohl auch Zulecg, NJW 1993, S. 31 (33).

$18 \mathrm{Vgl}$. dazu ausführlich Schröer, Die Kompetenzverteilung zwischen der EWG und ihren Mitgliedstaaten auf dem Gebier des Umweleschutzes, Berlin 1992, S. 10s ff.; Jarass, EuZW t99r, S. 530 jeweils mit umfassenden weiteren Nachweisen zum Diskussionsstand.

19 Scheuing, EuR 1989, S. 179; Pernice . VVwZ 1990, S. 203 m. w. N.

$20 \mathrm{Vgl}$. Schroer. (Fn. 18); Jarass (Fn. 18), S. $531 \mathrm{~m}$. w. N.

21 Abgedruckt in EuR 1991, S. 175 mit kritischer Besprechung von Everling. S. 179; vgl. zu dieser Auseinandersetzung Zuleeg, NJW $1993, \mathrm{~S}_{31}$ (32 f.).

22 Vgl. Epiney/Furrer, EuR 1992, S. 369 (394). 
Anwendungsbercich des Art. I 3 os EGV nur Richtlinien mit reinem oder doch ganz überwiegendem Umweltschutzcharakter. ${ }^{23}$ Deswegen, aber auch im Interesse der Rechtssicherheit, erscheint es überzeugender, wenn man im Anschluß an dic überwiegende Meinung im Schrifttum darauf abstellt, in welchem Politikbereich die jeweilige Regelung ihren Schwerpunkt findet: Art. 100a EGV ist als Rechtsgrundlage ciner Maßnahme dann heranzuzichen, wenn der Schwerpunkt der Regelung binnenmarktbezogen ist, insbesondere also dann, wenn zur Vermeidung von Wettbewerbsverzerrungen produktbezogene Normen harmonisiert werden. Demgegenüber sind produktionsbezogene Normen, deren umweltpolitische Ziele eine geringere Sachnähe zum Komplex des Abbaus von Handelshemmnissen aufweisen, auf die Rechtsgrundlage des Art. 1 zos EGV zu stürzen.

Ein über den ausdrücklichen gemeinschaftsrechtlichen Normenbestand hinausgehendes Grundprinzip des Gemeinschaftsrechts verkörpert der "Grundsatz des bestmöglichen Umweltschutzes«. In Zusammenschau verschiedener Vertragsbcstimmungen entwickelte dic Literatur im Anschluß an Zuleeg ${ }^{24}$ diesen Grundsatz als Handlungsanweisung für den Gemcinschaftsgesetzgeber sowie als Interpretationsregcl des Gemeinschaftsrechts. In Verbindung mit dem umweltrechtlichen Subsidiaritätsprinzip des Art. ı zor IV EWGV und Art. rooa IV EWGV wurde dieser Grundsatz auch als Kompetenzregel zugunsten der Mitgliedstaaten im Sinne einer "Schutzverstärkungsklausel « interpretiert. In der ncueren Literatur ist der Grundsatz des bestmöglichen Umweltschutzes vertiefend aufgenommen und fundiert aus dem gemeinschaftsrechtlichen Normenbestand hergeleitet bzw. bestätigt worden. ${ }^{\text {s }}$ Obwohl er im Schriftum zunehmend positive Würdigung erfährt ${ }^{26}$, ist er in der Praxis noch nicht anerkannt und bleibt auch in der Literatur umstritten. ${ }^{27}$

Der EUV brachte im Bcreich der Umweltpolitik cinige gesondert zu erwähnende Ergänzungen und Neuerungen. ${ }^{28}$

Entsprechend der erwähnten Erklärung des Europäischen Ratcs von Dublin hat die EG in dem neuen Art. 2 EGV ". . . e ein beständiges nichtinflationäres und umweltverträgliches Wachstum...« in ihren Aufgabenkatalog übernommen. Dieser Begriff muß aus systematischen Gründen im Sinne des Konzepts des "sustainable development " interpretiert werden. ${ }^{29}$ An einer näheren Definition des "concept of sustainibility" fehlt cs allerdings, so daß sich konkrete Folgerungen nicht schon aus dem Vertragstext ableiten lassen, sondern erst durch eine (systematische) Konkretisierung zu gewinnen sind. Dementsprechend nimmt das 5. Umweltaktionsprogramm der EG mit demTitel "Für eine dauerhafte und umweltgerechtc Entwicklung « (der cnglische Titel lautet "Towards Sustainable Development «) ${ }^{30}$ als konzeptioncllc Parallele zu den Dokumenten des UNCED-»Erdgipfels « von Rio de Janeiro vom Juni 1990 den dort verankerten Kernbegriff der "sustainibility «"' auf und versucht ihn in

23 Ebenso Jarass (Fin. 18 ).

24 Vgl. Zuleeg, NVwZ 1987, S. 280 (283 f.); ahnlich Scheuing, EuR 1989, S. 152 (167, 178 f.); Kramer, FuGRZ 1988, S. 28 s (288); Pernice, NVwZ 1990, S. 201 (206 f.); Haitbronner, in: Calliess/Wegener, Furopaisches Limweltrecht als Chance, 1992, S. $26 \mathrm{ff}$.

is Vgl. etwa Kahl, Umweltprinzip und Gemeinschaftsrecht, 1993, S. icfl. und ss ff.; stehe auch wicgand, DVBl 1993, S. 533 ff.; Epiney/Furrer, EuR 1992, S. 371 (388).

26 Vgl. nur Scheuing, I:uR 1989, S. 152 (: 67 ff.); Pernice, NVwZ 1990, S. 201 (206 ff.): Kramer, in: Renge-

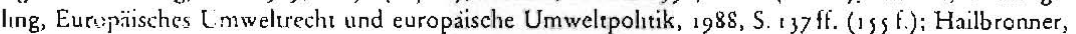
EuGRZ 1989, S. 101 (1171.); ders., in Calleess/Wegener (FN. 10), S. 15 (26 If.); Kahl, Umweltprinzip, S. icff. und 6y ff. jewels $\mathrm{m}$. $\mathrm{w} . \mathrm{N}$.

27 Vyl. Epincy/iurrer, E.uR :992, S. 369 (400 fi.) m. w. N.

28 Vgl. hierzu ausfuhrlich Epiney/Furrer, EuR 1992, S. 369 ff.; Ress, Vortrage (FN. 10), S. 3 ff.

$29 \mathrm{Vgl}$. Verhoeve/Bennet//Wilkinson, Maastricht and the Environment, Institute for European Environmental Policy, London 1992, S. 14 f.

30 Vgl, Dok. KOM (92) 23 snd.\% vom 3. 4. 1992, dazu Wagenbaur, EuZW 1993, S. 241.

31 Hierzu ausführlich Ruifert, ZUR 1993, S. 208 (209). 
der gemcinschaftlichen Umweltpolitik umzusetzen. ${ }^{32}$ Art. 3 lit. k EGV nimmt nunmehr auch die Umweltpolitik ausdrücklich in den Zielekatalog der EG auf. Damit hat die EG über den Art. 1 3 or EWGV hinaus eine "Staatszielbestimmung "Umweltschutz erhalten, die ausdrücklich als gleichrangig neben die anderen Ziele der EG tritt und insofern als wcitere Aufwcrtung der Umwcltpolitik in Geiste des erwähnten Dubliner Environmental Imperative $\approx$ zu verstehen ist. ${ }^{33}$

Interessant sind auch die Neuerungen im Entscheidungsverfahren, die die Stellung des EP aufwerten.

Abgeschen von den Ausnahmen in Absatz 2 wird in dem neuen Art. 130 E EGV die Mchrheitsentscheidung im Rat und das Verfahren der Zusammenarbeit (Art. I 49 II EWGV = neu Art. $189 \mathrm{c}$ EGV) im Hinblick auf das EP eingeführt. Hierdurch mag zum einen das Veto der den Umweltschutz bremsenden Staaten überwunden werden, zum anderen wird der Einfluß des EP, das eincm fortschrittlichen Umweltschutz vergleichsweise aufgeschlossen gegenübersteht, größer ${ }^{\text {`4 }}$. Eine bedeutcnde Ändcrung stellt insofern auch Art. I 30 III EGV dar, der für die erwähnten Aktionsprogramme das neue Verfahren des Art. 1896 EGV's einführt, welches dem EP erstmals eine Art Mitentscheidungsrecht ncben dem Rat einräumt.

Eines der ganz zentralen Probleme im Bereich der Umweltpolitik ist die zögerliche Umsetzung und mangelhafte Durchführung der mühsam zustande gekommenen EG-Gesetzgebung. So werden (auch in der Bundesrepublik) vicle EG-Richtlinien verspätet bzw. unzureichend umgesetzt. ${ }^{36}$ Überdies werden viele EG-Normen in der täglichen Praxis einfach umgangen oder nicht vollzogen. ${ }^{37}$ Selbst nach Urtcilen des EuGH wurden EG-Normen weiterhin mißachtet, da Konsequenzen in der Vergangenheit allenfalls auf der politischen Ebcne zu gewahren waren. Insofern bringt der neue Art. I7 II EGV cine interessante Neucrung, als in cinem eigenen Sanktionsverfahren im Falle der Nichtbefolgung eines Urteils der EuGH auf Antrag der Kommission die Zahlung eines Pauschalbetrags oder Zwangsgelds verhängen kann. Insofern stellt sich allerdings die Frage, wie die so verhängten $Z$ wangsgelder bcigetrieben werden sollen, da es diesbezüglich an einem entsprechenden Verfahren fehlt. Die eigentlich bessere Alternative, dem EuGH die Kompetenz zu geben, das Einfrieren von Zahlungen der EG, etwa aus dem Agrarfonds oder den Strukcurfonds, als Sanktion anzuordnen, hätte indes den Nachteil, daß eine solche Sanktion in der Regel keinerlei Verbindung zu der im Umweltbereich liegenden Vertragsvcrletzung haben würde. Rechtlich ließe sich eine Sanktion dieser Art auf die erwähnte Querschnittsklausel des Art. 1 zor II S. 3 EGV stützen. ${ }^{\text {\$ }}$

Angesichts dieser "Vcrtragslücke « hat der EuGH im Wege sciner neffet-utile«-Auslegung Sanktionsmöglichkeiten entwickelt: Nur vor diesem Hintergrund kann etwa dic Rechtsprechung des EuGH zur unmittelbaren Anwendbarkeit/Wirkung von nicht fristgemäß oder unzureichend durch die Mitgliedstaaten umgesetzten Richtlinien, die Rechte einzelner begründen und hinreichend bestimmt sind, verstanden werden. ${ }^{39}$

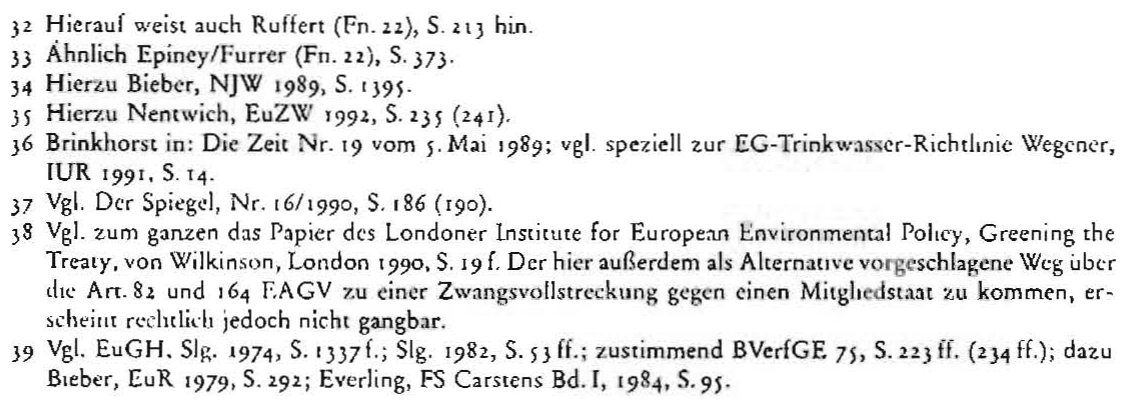


Dies gilt um so mehr für die neuere Rechtsprechung des EuGH, die in der Rechtssache "Francovich «' eine Art "gcmeinschaftsrechtliche Staatshaftung eingeführt hat, nach der ein Mitgliedstaat für die Vermögensschäden haftet, die dem einzelnen daraus erwachsen, daß cine Richtlinie nicht ordnungsgemäß in nationales Recht umgesetzt wurde. Diese Rechtsprechung wird sich in ihren Grundsätzen auch auf entsprechende Klagen wegen Nichtumsetzung von EG-Umweltrichtlinien übertragen lassen. "

Eine Hilfe bei der Vermcidung des skizzierten Vollzugsdefizits mag der neue Art. rзos V EGV sein, der bei "unverhälınismäßig hohen Kosten für die Behörden cines Mitgliedstaats « aufgrund einer umwcltschützenden Maßnahme eine finanzielle Unterstützung aus dem Kohäsionsfonds des Art. I zod EGV (Umwelt, Verkehrsinfrastruktur) vorsieht. Dem lag folgender Gedanke zugrunde: Umweltschutz verursacht Kosten und hemmt unter Umständen dic wirtschaftliche Entwicklung strukturschwacher Regionen und Mitgliedstaaten. Schon aus diesem Grunde wird im Ministerrat so manche umweltpolitische Maßnahme waus- oder abgebremse " bzw. später bei ihrer Umsetzung oder Durchführung in den Mitgliedstaaten leerlaufen gelassen. Gerade im Hinblick auf mögliche Mehrheitsentscheidungen im Ministerrat forderte u.a. Spanien, daß kostenträchtige EG-Unweltauflagen durch Finanzmittcl der EG aufgefangen werden. ${ }^{i^{2}}$

Um Befürchtungen einiger Mitgliedstaaten im Hinblick auf cin Absenken ihrer Schutzstandards entgegenzukommen, sicht der geänderte Art. I 30 II S. ${ }_{4}$ EGV bei Harmonisierungsmaßnahmen im Umweltschutz die Einfügung einer »... Schutzklausel vor, mit der die Mitgliedstaaten ermächtigt werden, aus... umweltpolitischen Gründen vorläufige Maßnahmen zu treffen..... Damit ist im Vertrag eine Möglichkeit eingefügt worden, dic es erlaubt, im Sekundärrccht unter bestimmten Voraussetzungen Schutzklauseln festzuschreiben, durch die die Mitgliedstaaten ermächtigt werden, primärrechtliche Grundsätzc zu derogicron. Insofern steht dem Gemeinschaftsgesetzgeber nunmehr ein Mittel zur Verfügung, den Bedürfnissen zur Lösung regionaler Umwcltprobleme entgegenzukommen. ${ }^{43}$ Im Unterschied zu den erwähnten inhaltlich unveränderten Schutzverstärkungsklauseln des Art. Iooa IV und 1 zot EGV, die fortschrittlicheren Staaten einen wcitcrgehenden Umweltschutz. ermöglichen wollen, dient die Schutzklausel der Abwendung oder Behebung umweltpolitischer Ausnahmesituationcn. Art. r zor II S. 4 EGV ähnelt insoweit den Art. I 2 ff. des Abkommens über den Europäischen Wirtschaftsraum (EWRV), wo dic Anwendung einer Notstandsklausel bei wökologischen Schwierigkciten « ausdrücklich anerkannt wird.44

Eine wichtige Neuerung stellt schließlich Art. I zor II S. I EGV dar, in dem einerseits analog zu Art. rooa III EWGV das Gebor der Verfolgung eines hohen Umweltschut\%niveaus festgeschrieben wird, andererseits ein Gebot der Berücksichrigung der unterschiedlichen Gegebenheiten in den einzelnen Regionen der Gemeinschaft verankert ist. Letzteres trägt der Erkenntnis Rechnung, daß Umweltpolitik sich an den national, regional und lokal stark differierenden Umweltbedingungen und -problemen orientieren muß, entspricht also dem Gebot der Differenzierung. 45 Jenes ist in Einklang mit dem Gebot eines hohen Schutzniveaus zu bringen.

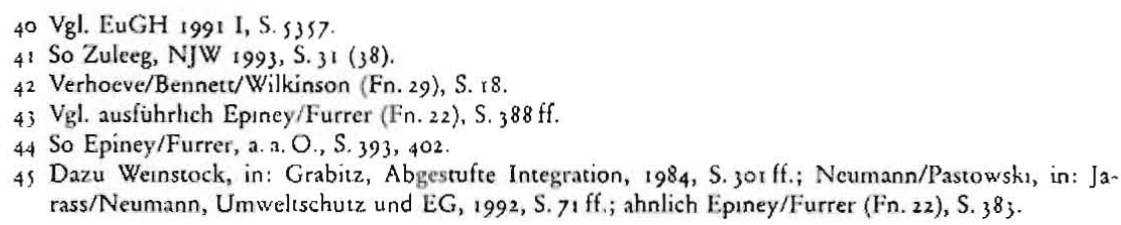


Zentrale Bcdeutung kommt der Frage zu, wie sich nationale Maßnahmen im Bereich des Umweltschutzes zum Binnenmarkt verhalten. Insofern ist zu unterscheiden, ob die EG bercits durch Harmonisierungsmaßnahmen regclnd tätig geworden ist oder nicht.

Im letzteren Falle sind die Anforderungen den Art. 30 ff. EGV über den freien Warenverkehr zu entnehmen, im ersteren Fall sind die Art. rooa IV oder I zot EGV zu prüfen.

Die materiellen Regelungen in Art. 30 und 34 EWGV untersagen mengenmäßige Ein- und Ausfuhrbeschränkungen sowie Maßnahmen gleicher Wirkung. Ausnahmen sind nach Art. 36 EWGV zum Schutz der Gesundheit und des Lebens von Menschen, Tieren oder Pfianzen, zum Erhalt nationalen Kulturguts und aus Gründen der öffentlichen Ordnung und Sicherheit zulässig.

Da ausdrückliche mengenmäßige Beschränkungen der Ein- und Ausfuhr in der Praxis kaum noch vorkommen, hat sich die Rechtsprechung des EuGH früh auf Maßnahmen gleicher Wirkung konzentriert. Nach sciner grundlegenden Dassonville-Entscheidung ist eine Maßnahme gleicher Wirkung „jede Handelsregelung der Mitgliedstaaten, die gecignet ist, den innergemcinschaftlichen Handel unmittelbar oder mittelbar, tatsächlich oder potentiell zu behindern « ${ }^{46}$ Unter diese sehr weite Dcfinition ${ }^{47}$, die der Gcrichtshof vor allem zur Stärkung des Gemcinsamen Marktes entwickelt hatte, fallen strengere nationale Umweltworschriften grundsätzlich selbst dann, wenn sie auf inländische wic ausländische Erzeugnisse unterschiedslos anwendbar sind und daher keine dirckte Diskriminierung ausländischer Produkte darstellen..$^{8}$ Seine ursprüngliche Ausdehnung der Verbote der Art. 30 und 34 auf unterschiedslos anwendbare, also nicht diskriminierende Maßnahmen hat der EuGH in seinem "Cassis de Dijon «-Urteil ${ }^{49}$ eingcschränkt, indem er crklärte, sog. "zwingende Erfordernisse « könnten solche Maßnahmen bereits auf der Tatbestandsebene rechtfertigen. In seiner grundlegenden "Dänischen Pfandflaschenentscheidung ${ }^{\text {s० }}$ hat der Gerichtshof diese These wieder aufgegriffen und den Umweltschutz unter Hinwcis auf die Stellung des Umweltschutzes in den Art. s zorff. EWGV in den Katalog der zwingenden Erfordernisse aufgenommen. ${ }^{\prime \prime}$ Im Hinblick auf die Frage der Rechtfertigung von Handelsbecinträchtigungen nach Art. 30 EGV ist zu prüfen, ob die mitgliedstaatliche Regelung nach den Maßstäben der "Cassis-Rechtsprechung " und der "dänischen Pfandflaschenentscheidung "s" unterschiedslos auf ausländische und inländische Produkte answendbar ist, also kcine (vcrschlcierte) Dis-

${ }_{46} \mathrm{EuGH}, \mathrm{Slg} .1974$, S. $837,852$.

47 Siehe dazu aber auch neuerdings das Urtell in Fall Keck, EuZW 1993, S. 770; dazu Ress, EuZW 1993, S. 745 .

48 So gelten beispielsweıse die Ausnahmen fur Katalysatorwagen, die verschiedene Smog-Verordnungen deutscher Lander vorsehen, selbstverstandlich in gleicher Weise fur deutsche wic fur franzosische Katalysatorwagen. Dennoch stellten diese Regelungen nach Ansicht der Kommission ein unzulassiges Handelshemmnis dar, da der Katalysator in anderen Staaten der Gemeinschaft noch nucht im selben Umfange eingefuhrt seı wie in der Bundesrepublık, so daß auslandischen Herstellern insoweit Wettbewerbsnachteile entstehen konnten; vgl. Begrundete Stellungnahme der Kommission vom 16. 12. 1988, K (88) 2274; dazu Hailbronner, EuGRZ 1989, S. เо1.

$49 \mathrm{EuGH}$, Slg. 1979, S. 649, 662.

so EuGH, Urteil v. 20.9.1988, NVwZ 1989, S. 849 f.

st Zu dieser Entwicklung Rengeling/Heinz, JuS 1990, S. $61_{3}$ ff.; neuerdings Kramer, CMLR 1993, S. « । ff.; Lueder, Z.UR 1993, S. $169 \mathrm{ff}$

S2 EuGH Slg. 1979, S. 649 und Slg. $1988, S_{4} 607$. 
kriminierung darstellt, ob sie tarsächlich dem Umweltschutz. dient und verhältnismäBig ist. 53

Im Rahmen der Verhältnismäßigkeitsprüfung ist nach der Rechtsprechung des EuGH eine Güter- und Intcresscnabwägung zwischen Umweltschutz und freiem Warenverkehr vorzunehmen. ${ }^{5 "}$ Diesbezüglich wird mit guten Gründen in der neueren Literatur Kritik geübt: Die Verhältnismäßigkeitsprüfung, die der EuGH in der "dänischen Pfandflaschenentscheidung" für umweltrelevante nationale Maßnahmen durchführe, nehme den Mitgliedstaaten die Möglichkeit, im Rahmen ihrer verbliebenen Restkompetenz, bei handelsrelevanten Regelungen in bezug auf den Umweltschutz selbst über das von ihnen angestrebte Schutzniveau zu entscheiden. Denn die Mitgliedstaaten hätten sich auf ein "vernünftiges", mithin verhältnismäßiges Schutzniveau zu beschränken, das den innergemeinschaftlichen Handel am wenigsten cinschränke. Mithin sei es ihnen verwehrt, eincn ihren Ausgangsbedingungen entsprechenden optimalen Umweltschutz zu verwirklichen."ss

Dennoch ist nicht zuletzt dem oft mißverstandenen "Wallonien-Urteil « vom 9. Juli 1992 des LuGH ${ }^{56}$ eine T'endenz zu entnehmen, dem Umweltschutz im Verhältnis zum freien Warenverkehr eine stärkere Stellung einzuräumen. So stellt der Gerichtshof im Rahmen der Prüfung des Grundsatzes der Verhältnismäßigkeir für die Frage, ob das belgische Importverbot von Haushaltsabfällen zum Schutz der Umwelt gerechtfertigt ist, auf das "Ursprungsprinzip« des Art. I 3or II S. I EWGV ab. Damit macht der EuGH deutlich, daß Art. I 3 or EWGV Leitlinien für die umweltverträgliche Ausübung der Marktfreiheiten aufstellt, die auch bei der Beurtcilung der Zulässigkeit mitgliedstaatlicher Schutzmaßnahmen herangezogen werden müssen. ${ }^{77}$ Wenn in Art. Izor I EGV von einer Verbesserung der Umweltqualität gesprochen wird und in Art. $100 a$ III FGV von einem Binnenmarkt mit hohem Umweltschutzniveau ausgegangen wird, dann muß die Güter- und Interessenabwägung im Rahmen des Verhältnismäßigkeitsprinzips bei Art. 30 EGV dynamisch vorgenommen werden, so daß die Mitgliedstaaten nicht darauf beschränkt werden, nur das bestehende Umweltschutzniveau zu halten. ${ }^{8}$ Nach dem Urteil ist überdies die Diskriminierung anhand der Auswirkungen einer Maßnahme auf die Unwelt zu definieren. Daher ist nicht jede Ungleichbehandlung der Waren aus anderen Mitgliedstaaten schon eine verbotene Diskriminierung. 59

Etwas andere Anforderungen gelten für die von einer Harmonisierungsmaßnahme abweichende mitgliedstaatliche Schutzvcrstärkung. Nach Arr. 10oa IV S. I und 2 EGV muß diese durch wichtige Erfordernisse im Sinne des Art. 36 EGV oder des Schutzes der Umwelt gerechtfertigt sein. Dabei darf sie kein Mittel zur willkürlichen Diskriminierung und keine verschleiertc Beschränkung des Handels zwischen den Mitgliedstaaten darstellen. Der Umweltschutz, der nach der Rechtsprechung als "zwingendes Erfordernis « im Rahmen des Art. $30 \mathrm{EGV}$ anerkannt ist, wird hier in die Dogmatik des Art. 36 S. 2 EGV eingeordnet. ${ }^{60}$ Folglich darf in Art. $100 a$ IV EGV, insoweit im Unterschied zu Art. $30 \mathrm{EGV}$, die Maßnahme diskriminierend sein, dies

i3 Ausfuhrlucher Übirhlick anhand der Rechtsprechung bei Lueder, ZUR 1993. S. 165 (167(f)); Kramer, F.T.C-Triaty and Finvironmental Protection, S. 35 If.; Pernice, NVwZ 199\%, S. 209.

S4 Beispucie bei Zulecs, NJW 1993 , S. 34 f,; sowic Lueder (Fn. 51), S. $168 \mathrm{i}$.

ss So Kramer, CMLR 1993, S. 111 (120 iff.).

s6 Abgedruckt in EuZWW 1992, S. 577; dazu v. Wilnowsky, EuR 1993, S. 414 ff.

s7 Vgl. v. Wilmowsky ; (n. 56), S. ${ }_{14}$ f; Zuleeg, NJW 1993, S. 34.

s8 So auch v. Wilmowsty, ebenda.

59 So Zulceg (Fn. 57), S. 35 unter Berufung auf Rz. 34 des Urtells; austuhrtich v. Wilmowsky (Fn. 56), S. 4 ist.

60 Vgl. I angeheine, in: (irabitz, Kommentar zum E.WG-Vertrag, Ar. ısa, Rn. 72; Pernicc, NVwZ 1990, S. 2:7: wohl auch Hailbronner, in: Calluss' W'egener (Fn ro), S. 27 f. 
aber nicht willkürlich, so daß sachgerechte Gründe für eine unterschiedliche Bchandlung ausländischer und inländischer Produkte bestehen müssen. "' Entscheidend kommt es dann wic bei Art. zo F.GV auf dic Güter- und Interessenabwägung im Rahmen des Grundsatzes der Verhältnismäßigkeit an. ${ }^{62}$ Zum Teil wird dic Auffassung vcrtreten, daß eine Rechtfertigung der Schutzverstärkung in den nach Art. $100 a$ EGV harmonisierten Bereichen »deutlich strengeren Anforderungen « unterliegen müsse, als dort, wo eine Umweltgesetzgebung auf EG-Ebene noch nicht vorliege. ${ }^{63}$ Hiergegen spricht allerdings unter teleologischen Auslegungsgesichtspunkten, daß sich gerade in Art. $100 a$ IV EGV zeigt, daß den dort genannten Rechtsgütern ein höherer Stellenwert als der Vollendung des Binnenmarktes beigemessen wird. ${ }^{64}$ Hierfür spricht auch die in der Einführung dargelegte umweltfreundliche Tendenz der EEA, die durch den EUV/EGV noch unterstrichen wird. ${ }^{6 s}$

Gem. Art. 1 3ot S. 2 EGVmüssen schutzverstärkende Maßnahmen der Mitgliedstaaten mit dem "Vertrag vereinbar" sein. Was dies konkret bedeutet, ist in der Literatur umstritten. Zum Teil wird die Ansicht vertreten, daß nicht gegen die Art. 30, 100 und 100a EWGV verstoßen werden dürfe, es sei denn, daß die Voraussetzungen des Art. 36 EGV, der "Cassis-Rechtsprechung " oder des Art. xooa IV EGVvorliegen. ${ }^{66} \mathrm{Nach}$ anderer Meinung soll dieser Satz als Verweis auf die allgemeinen Prinzipien des Gemeinschaftsrechts wie den Grundsatz der Verhältnismäßigkeit, die Gemeinschaftstreue und insbesondere auch auf die Zielsetzungen des Art. 13 or EGV zu verstehen sein. ${ }^{67}$

\section{Schlußfolgerungen}

Die EG ist trotz interessanter Ansätze und zum Teil durchaus fortschrittlicher Normen im Umweltschutz noch weit davon entfernt, eine ökologische Trendwende im Binnenmarktkonzept eingeleitet zu haben. Zunächst ist das EG-Umweltrecht trotz aller Bekundungen insbesondere dort fortschrittich, wo es eher unverbindliche politische Zielvorgaben enthält, die keinen oder nur wenig rechtlich-verbindlichen Gehalt haben. Die Neuerungen des FUV im Hinblick auf die Art. I zorff. EGV stellen eher eine Abstimmung und Anpassung an die Vorschrift des Art. 100 a EV dar. Die durchaus fortschrittlichen Zicle und Grundsätze können allenfalls mittelbar rechtliche Auswirkungen entfalten. Die Rolle des EP wird nur im Bercich des »softlaw « der Aktionsprogramme durch Mitentscheidung gestärkt. Die Ansätze zur Beseitigung des Vollzugsdefizites bleiben bescheiden, da das Sanktionsverfahren noch einmal fast von gleicher Dauer wie das Urteilsverfahren sein dürfte und damit säumigen Mitgliedstaaten noch einmal Jahre Zcit verschaff. Inwiefern die Fondsgelder den Vollzug des Umweltrechts fördern werden, bleibt abzuwarten.

Überdies trägt das gemeinschaftliche Umwcltrecht den verschiedenen ökologischen und wirtschaftlichen Ausgangsbedingungen in den zwölt Mitgliedstaaten zu wenig Rechnung. Insofern bietet das binnenmarktbezogene europäische Umweltrecht nach wie vor zu wenig Spielraum für diffcrenzierte, flexible, rcgional angepaßtc Konzepte im Unweltschutz.

61 A. A. Scheuing, EuR 1989, S. 169 f.; Grabizz, in: Grabitz, Kommentar zum EWG-Vertrag, Art. 130r, Rn. $17 \mathrm{~m}$. w. N., der zusatzlich auf die oben erwahnte "Cassis-Rechtsprechunge abstellt.

6. Vgl. nur Hailbronner, un: Calliess/Wegener (Fn. 10), S. 27; Pernice (Fn. 60), S. 208, der hier das Prinzip der prakeisehen Konkordanz von Hesse einfuhrr.

63 So Pernice, cbenda in. w. N.

64 Vgl. insoweit Grabıtz, in: Grabitz (Fn. 61), Art. 130t, Rn. 17; Zuleeg, NJW 1993, S. 35.

6s So auch Epincy/Furrer, EuR 1992, S. 401.

66 Lietzmann, in: Rengeling, Europaisches Umweltrecht und europaische Umweltpolitik, 1988, S. 175.

67 So Epiney/Furrer, EuR 1992, S. 4 C2. 
Eine Ausnahme von der Flucht in das "Beruhigungsrecht « könnte die neu gefaßte Querschnittsklausel sein, deren rechtsverbind licher Charakter in der konkreten Formulierung deutlich zutage tritt. Mit ihrem integrierenden Ansatz stellt sie, jedenfalls theoretisch, einen bedeutenden Ansatz. hin zu einem ökologischen Rechtssystem dar. Wenig Hoffnung macht allerdings der Umgang mit der bisherigen Querschnittsklausel in der Praxis, die sogar vom Umweltministerrat gerügt wurde. ${ }^{68}$ Entscheidend wird damit die Frage ihrer Justiziabilität; die zurückhaltenden Stimmen in der Literatur lassen aber auch insofern auf wenig Fortentwicklung hoffen.

\section{Aspekte eines Umbaus der EG/EU zu einer ökologischen Rechtsgemeinschaft}

Um den Herausforderungen der eingangs skizzicrten schlcichenden Umweltkatastrophc in der Risikogesellschaft adäquat begegnen zu können, muß das derzeitige Rechtssystem, zu dem das der EU gehört, in einem ökologischen Sinne fortentwikkelt werden. Eine solche Fortentwicklung wird derceit unter den Stichworten "Umweltstaat ${ }^{69}$ und "ökologischer Rechtsstaat ${ }^{70}$ diskutiert. Entsprechend licßc sich mit Blick auf die EU/EG das Ziel einer ökologischen Rechtsgemeinschaft postulieren, denn die EU/EG hat sich bereits über eine Wirtschaftsgemeinschaft hinaus zu einer Rechtsgemeinschaft entwickelt. ${ }^{71}$ Unter einer ökologischen Rechtsgemeinschaft kann ein Rechtssystem verstanden werden, das umfassend ökologisch ausgerichtct ist. Dics meint insbesondere eine Rechtsurdnung, die ökonomisches Handeln konsequent auf Umweltverträglichkeit programmiert, die versucht, von vornherein umweltverträgliches Verhalten, insbesondere umweltverträgliche Produktionsverfahren, zu etablieren, und die den sektoralen Ansatz im Umweltrecht zugunsten cines integrierenden, intermedialen Ansatzes aufgibt ${ }^{72}$. Ein solches programmatisches Unionsziel bedarf der Konkretisicrung und Ausgestaltung. Einige Elemente finden sich bereits im Gemeinschaftsrecht bzw. sind in diesem bereits angelegt, so daß es nur einer konsequenten Fortentwicklung bedarf. Andere Elemente müßten crst neu geschaffen werden.

\section{Vorsorgeprinzip und Vorrangklausel (in dubio contra projectum)}

Auch wenn der geänderte Art. I zor II S. z EGV dic EG-Umwcltpolitik noch deutlicher als bisher auf den "Grundsatz der Vorsorge und Vorbeugung "verpflichtet, beläßr der Vertrag diesen maßgeblichen Grundsatz inhaltlich und instrumentell ohne nähere Konturen. Damit bleibt er ohne hinreichende rcchtliche Verbindlichkeit. $^{73}$

Ein ökologisches Rechtssystem muß abcr zuvorderst das grundlegende Problem der Risikogesellschaft zu lösen versuchen: die Kluft zwischen der Macht des technolo-

68 Informeller Rat von Gleneaglus im September 1992, Conclusions of the Presidency.

69 Ladenburger Kolleg "Umweltstaat «; dazu Kloepfer, DVBI 1994, S. 12; zum Sehlußkongreß vgl. den Tagungsbericht von A. Lubbe, ZUR 1994, S.42.

70 Tagung "Wege zum okologischen Rechtsstaat* des Insututs fur Umweltrel:t, Bremen, 6./7. 2. 1993; dazu Tagungsbericht von Retter, ZUR 1993 , S. 190.

71 Zur EG als Rechtsgemeinschaft vgl. neuerdings Zuleeg, NJW 1994, S. 545

72 Definition in Anlehnung an Murswiek, FAZ vom 26. 10. 1992, S. $3^{*}$

73 Hierzu ausfuhrlucher Verhoeve/Bennett/Wilkinson (Fn. 29), S. i7. 
gischen Handelns einerseits und der Ohnmacht des prognostischen Wissens von seinen Fernwirkungen andererseits. ${ }^{74}$ Insofern ist es nützlich, dic Nahprognose, mit der die betreffenden Projekte in der experimentell-technologischen Mikroperspektive angesetzt werden, und die Fernprognose, die sich auf die möglichen zukünftigen Folgen in der Ökosphäre bezieht, zu unterscheiden. Dabei ist die Sicherheir, die die Nahprognose hat, bei der Fernprognose unmöglich. Der verbleibenden Unwissenheit müßte durch das Prinzip des "Vorrangs der schlechten vor der guten Prognose“ Rechnung getragen werden."

Ziel eines insofern nötigen "Risikomanagements « ist die allgemeine Reduzierung des Besorgnispotentials. Vor dem I-Hintergrund der Irreversibilität vieler ökologischer Auswirkungen ist das Prinzip von "trial and error " fragwürdig geworden, so daß auf bestimmte Risikopotentiale unter Umständen verzichtet werden mu $\beta^{76}$, gewissermaßen also dic "Nulloption " denkbar werden muß.

Rechtlich bedeutet dies, daß das Vorbeugeprinzip durch einc Bewcislastverteilungs-

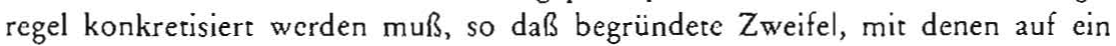
sogenanntes Restrisiko hingewiesen wird, durch zureichende Gründe entkräftet werden können müssen. Andernfalls muß die Vorrangklausel cingreifen, nach der dem Grundsatz "in dubio contra projectum "zu folgen ist. Die konkrete Durchführbarkeit dieses abstrakten Prinzips ist an die "Institutionalisierung konkreter Diskurse $^{77}$ (Kooperationsprinzip ${ }^{78}$, Mediation und Konfliktmittelung ${ }^{79}$, Beteiligungsrechte) geknüpft.

Wurde in der Literatur schon auf der Grundlage der gegenwärtigen Rechtslage ein grundsätzlicher Vorrang des Umweltschutzes im Konfliktfall zugesprochen ${ }^{80}$, so muß gerade eine ökologisch effektivc Reform des I:GV diese Überlegung miteinbeziehen. So wäre es denkbar, Art. r zor um eine "Vorrangklausele des Inhalts zu ergänzen, daß der "Konfliktfall Ökonomic - Ökologie" im Sinne des obigen »in dubio contra projectum " präjudiziert wird, wenn sowohl ein "Gleichgewicht $\approx$ der Argumente herrscht, als auch mit hoher Wahrscheinlichkeit erhebliche (irreparable) Umweltschäden zu erwarten (Vorbeugungsargument) sind. Sollte die Vorrangklausel nicht beachtct werden, so könnte man dem Gemeinschaftsgesctzgeber vor dem $\mathrm{EuGH}$ einen schwerwiegenden Abwägungsfehler anlasten, der unter Umständen zur Aufhebung der Maßnahme führt. Die "Vorrangklausel « in Art. I zor EGV würde nicht nur in Verbindung mit der Staatszielbestimmung, sondern auch in Verbindung mit der "Querschnittsklausel « des strenger gefaßten Art. I 3 or II S. 3 EGV, alle Politikbereiche der EG erreichen und prägen.

Für den Bereich des Sekundärrechts ergäben sich hieraus beispielswcise folgendc Konsequenzen: Die Umweltverträglichkeitsprüfung (UVP) als das Instrument des vorbeugenden Umweltschutzes muß obigen Grundsätzen angepaßt werden. Die EG hat im Jahre 1985 die schon erwähnte UVP-Richtlinie verabschiedet, die allerdings im Laufe der langen Verhandlungen zunehmend verwässert worden war. ${ }^{81}$ Will man eine wirklich effektiv vorbeugende europäische Umweltpolitik betreiben, müßte die UVP-Richtlinie unter ökologischen Gesichtspunkten überarbeitet werden, um als

74 Vgl. Böhler, Deutsche Zeitschrift fur Philosophic, Heft 9/1991, S. 999 (1013); ders.; neuerdings in ZRP 1993, S. 389 (392).

75 Vgl. Jonas, Das Prinzip Verantwortung, Frankfurt a. M. 1984, S.6r ff.; Bohler (Fin. 74), S. 1013.

76 Rituer, DOV 1992, S. 641 (648f.).

77 In Anlehnung an Böhler (Fn. 74), S. 1014

78 Dazu Schrader, DÖV 1990, S. $326 \mathrm{ff}$.

79 Dazu ausfuhrlıch die Beitrage von Holznagel, Struegnicz, Benz, Kellermann, Ahrens, Fiuhr in IUR 1992, S. $37 \mathrm{ff}$.

80 Scheuing, EuR 1989 , S. 176, m.w. N.

81 Cupci, Umweltvertraglichkeitsprufung, 1986, S. 103 
cffektives Prüfungssystem im Sinne des vorbeugenden Umweltschutzes wirken zu können. ${ }^{82}$ Dem Gebot der Frühzeitigkeit entspräche es, die UVP auf Planung und Gesetzgebung auszudehnen. Das Ergebnis der UVP sollte zudem in der behördlichen Entscheidung über die Zulässigkeit einer umwcltrelevanten Maßnahme hinreichenden Einfluß haben, also nicht lediglich nur "berücksichtigt« werden. Die sog. "Null-Option«, d.h. der Verzicht auf das Projekt, sowie eine "Vorrangklausel $\propto$ im Sinne eines »im Zweifel für die Umwelt « sind insofern unabdingbar. Darüber hinaus muß angesichts der krassen Umsetzungsdefizite der UVP in der täglichen Praxis ${ }^{{ }_{3}}$ eine mit den erforderlichen Kompetenzen ausgestattcte Kontrollinstanz eingerichtet werden.

In Zusammenhang mit dem Vorsorgeprinzip steht die Umwelthaftung ${ }^{8_{4}}$. Verschärfte Umwelthaftung, in Japan und den USA Realität, zwingt zur Vorsorge und ist damit ein effizientes Mittel der Umwcltpolitik. Gecigneter und in der aktuellen Diskussion häufig betonter Anknüpfungspunkt ist die Versicherbarkeit ${ }^{85}$ von Umweltrisiken ${ }^{86}$. Viele Zukunftsindustrien arbeitcn derzeit jenseits der Versicherungsgrenze. Der fehlende private Versicherungsschutz für umweltrelcvante und risikobehaftete Tärigkeiten wäre ein geeignetes Instrument, bci dem Selbstkontrolle und Sclbstbegrenzung incinandergreifen.

Im Haftungsrecht sollten anstelle strikter Kausalitätsnachweise festzulegende Indikatoren (z.B. über die Gesundheitsgefährlichkeit bestimmter Abfallstoffe) zur Grundlage der rechtlichen Anerkennung von Schädigungen gemacht und dic Beweislasten umverteilt werden, so daß die betrieblichen und wissenschaftlichen Aktcure rechtfertigungspflichtig werden. Wenn in diesem Sinne "im außervertraglichen Haftungsrecht künftig alle wichtigen Schädigungspotentiale durch Gefährdungshaftungen abgedeckt « würden, bedeute dies einen Wechsel von der Verschuldens-zur Risikozurechnung und damit auch einen Wechsel von der Schadenszurechnung zur Schadensverteilung: "Denn tatsächlich sind Gefährdungshaftungen - in Verbindung mit Haftpflichtversicherungen - nichts andercs als Schadensverteilungssysteme., ${ }^{87}$

Parallel hierzu wandclt sich die Anschauung vom Zweck des Haftungsrechts vom Schadensausgleich zur Schadensprävention. So wird gerade im Bereich der Gefährdungshaftung zunehmend die Präventivfunktion des Haftungsrechts als gleichrangig, wenn nicht gar als vorrangig im Verhältnis zur Schadensausgleichsfunktion gewertet. $^{88}$

Die Bundesrepublik hat mit dem am I. Januar 1991 in Kraft getretenen Umwelthaftungsgesetz diesen Weg der verschärften Umwelthaftung beschritten." Auf EGEbene hat die Kommission einen Vorschlag für eine Richtlinie über die zivilrechtliche Haftpflicht für Schäden, verursacht durch Abfall, vorgclegt. ${ }^{\circ 0}$ Unter dem Gesichtspunkt des in Art. r 3 or EGV festgeschriebenen "Verursacherprinzips « soll die EG-Richtlinie den Ausgleich von Schäden an Personen oder Sachen zugunsten des Opfers erleichtern, indem das Verschuldensprinzip aufgegeben wird, Beweiser-

82 Hierzu Bunge, DVBl 1987, S. 819 ; vgl. auch Jarass, NuR 1991, S. 201.

83 Vgl.: Der Spregel, Nr. 16/1990, S. 186 (193).

84 Hierz.u ausfuhrlich Diederichsen, Festschrift fur Rudolf Lukes, 1989, S. 41, wie auch Marburger, AcP 192 (1992), S. 1 ; mit Blick auf das UGB vgl. auch Enders/Reiter, VersR 1991, S. 1329.

8s Hierzu Schmidt-Salzer, VersR 1992, S. 793; Peter, IUR 1992, S. 79 .

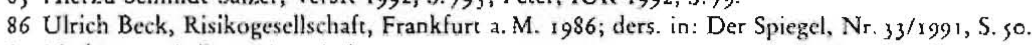

87 Marburger, AcP 192 (1992), S. 1 (29).

88 Vgl. Wagner, JZ 199r, S. i7s (r76) m. w. N.; Marburger (Fn. 84), S. 30.

89 Hierzu Schmidt-Salzer, VersR r991, S.9.

$90 \mathrm{ABl}$ l:C, C 192 von 23. 7. 1991, S.6; dazu Salje, IUR 1992, S. 66 (68 ff.) m. w. N.; vgl. neuerdıngs auch das „Grunbuchi der I:Ci-Kommission zur Haftung fur Umweltschaden, KOM (93) 47, Abl EG C 1993. Nr. 149, S. 12. 
leichterungen ermöglicht werden, eine umfassende Absicherung aller Arten von Schäden und Umweltbeeinträchtigungen erfolgen soll sowie Klagemöglichkeiten für Umweltverbände und eine Pflichtversicherung eingeführt werden.

\section{Ausdifferenzierung des Schutzniveaus ${ }^{91}$}

Die Umweltverbände schlagen in ihrem Report ${ }^{92}$ eine Neufassung des Art. Iooa Abs. 3 EWGV vor, die die Kommission verpflichten soll, in ihren Vorschlägen von dem »höchsten Schutzniveau im Umweltschutz, das in einem der Mitgliedstaaten besteht ${ }^{93}$, auszugehen. Auf den ersten Blick scheint dieser Vorschlag einem effektiven Umweltschutz dienlich, verhindert er doch ein Absenken des Schutzniveaus für "fortschrittliche" Staaten und verpflichtet die übrigen Mitgliedstaaten, das entsprechende Schutzniveau zu übernehmen. Auf den zweiten Blick dürfte eine solche Norm dem Umweltschutz aber eher hinderlich sein. Inzwischen setzt sich immer mehr die Einsicht durch, daß die Ausgangsbedingungen für den Umweltschutz in den Mitgliedstaaten und ihren Regionen in bezug auf ihre ökonomische Entwicklung, ihre geographische Lage, ihre Besiedelungsdichte, die ökologischen Verhältnisse und das Umweltbewußtsein der Bevölkerung viel zu unterschiedlich sind, als daß die Umweltprobleme Europas nach einheitlichen Konzepten samt einheitlichem (hohem) Schutzniveau gelöst werden könnten. Daher ist es nur verständlich, wenn im Ministerrat keine Einigung über Umweltschutz auf einem hohen Schutznivealı erzielt werden kann. Die Ausgangsbedingungen für Umweltschutz sind in Dänmark und Spanien einfach zu verschieden. Diese Tatsache gilt es zu akzeptieren, um auf dieser Basis dann nach Lösungen zu suchen. Der Formulierungsvorschlag des Reports begründet somit dic Gefahr einer Entscheidungsblockade im Sinne eines "Ganz oder gar nicht«, indem die Möglichkcit einer Kompromißlösung auf einem niedrigeren Schutzniveau und damit die Chance, eine europaweitc Lösung für das entsprechende Umweltproblem zu finden, verbaut wird.

Vor diesem Hintergrund läßt sich idealtypisch ein Gegenkonzept einer Kompetenzausübung im Umweltschutz entwickcln, das EG-weite Regelungen ermöglicht und glcichzeitig notwendige nationale und regionale Differenzierungen im Sinne eincr Schutzverstärkung erlaubr, indem entsprechende Handlungsspielräume für die dezentralen Regelungsebenen verbleiben. Danach solltc cine EG-Regclung nur einen Mindeststandard festsetzen, der es den Mitgliedstaaten und den Regionen ermöglicht, unter bestimmten Voraussetzungen notwendige schutzverstärkende Maßnahmen beizubehalten, aber vor allem auch neu einzuführen. Ebenso müßten die Regionen im Hinblick auf den jeweiligen Mitgliedstaat und die EG befugt sein, jene strengeren, weitcrgehenden Maßnahmen legislativer wie administrativer Art zu ergreifen, die die spezielle regionale Umweltsituation erfordert. ${ }^{94}$

Diesem Konzept entspricht die Erkenntnis, daß sich in einem ökologischen Rechts-

91 Dazu ausfihrlich Calliess, in: Evers (Hrsg.), Chancen des Foderalismus in Deutschland und Furopa, Schriftenreihe des Deutschen Instıtuts fur Föderalismusforschung, Baden-Baden 1994, S. 173 ff.; Trittin, in: Calliess/Wegener, Europaisches Umweltrecht als Chance, 1992, S. 5 i If.

92 -Greening the Treaty * von WWF, CEAT, EEB, Brussel, November 1990

93 Ubersetzung des Verfassers aus dem Englischen.

94 Dazu ausfuhrlich Callıess, in: Evers (Fn.91), in diesem Sinne auch Tritin (Fn. 91), S. s6; ahnlich auch Brinkhorst, Subsidiarity and European Environnent Policy, Presentation to the First Jacques Delors Colloquium, Maastricht, 22 March 1991, S. 7, wobei allerdings unklar bleibt, ob der Begriff "mstruments * dic Schutzverstarkungskompetenz der dezentraleren Handlungseinheiten in obigem Sinne mitunafassen soll. 
system dic wesentlichen Entschcidungen in der Hierarchie der Rechtsquellen von oben nach unten verlagern. Dabei erfolgt die inhaltliche Auffüllung der gesetzlichen Tatbestände im Vollzug, der damit zu einer "normnachschöpferischen* Phase wird.9s

Die Möglichkeit des "opting-up« bietet der EGV schon jetzt auf der Grundlage des oben erwähnten, durch Auslegung ermittelten Grundsatz des bestmöglichen Umweltschutzes. Allerdings ist dieser Grundsatz noch nicht zum europarechrlichen Allgemeingut geworden. Um die kontroverse Diskussion über das "opting up" im Rahmen des Art. 100a Abs. 4 und Art. $130 t$ EGV ${ }^{96}$ zu beenden, schlägt der "Greening the Treaty «-Report der europäischen Umweltverbände die Neufassung des Art. rooa Abs. 4 EWGV vor, die eindeutig die "opting-up «-Möglichkeit festschreibt. Letztere ließe sich vielleicht noch besser unter Einbeziehung des Grundsatzes des bestmöglichen Umweltschutzes formulieren.

In diesem Zusammenhang erscheint auch der Umgang mit dem Subsidiaritätsprinzip des Art. 1 zor Abs. 4 EWGV von Bedeutung. Völlig unberührt von der Dezentralisierungsforderung der Umweltbewegung und der akcuellen Subsidiaritätsdiskussion in der EG" , läßt der Report der Umweltverbände die "Besserklausel« des Art. I zor Abs. 4 EWGV unverändert und spricht der EG eine Generalkompetenz im Umweltschutz zu, die durch einige Aufgabenzuweisungen konkretisiert wird. Durch den neuen Art. $3 \mathrm{~b} \mathrm{EGV}{ }^{8}$ wirkt das Subsidiaritätsprinzip als allgemeines Rechtsprinzip nunmehr ausdruicklich - in die gemeinschaftliche Umweltpolitik hinein:

"Die Gemeinschaft wird innerhalb der Grenzen der ihr in diesem Vertrag zugewiesenen Befugnisse und gesetzten Ziele tätig.

In den Bereichen, die nicht in ihre ausschließliche Zuständigkeit fallen, wird dic Gemeinschaft nach dem Subsidiaritätsprinzip nur tätig, sofern und soweit die Ziele der in Betracht gezogenen Maßnahmen auf Ebene der Mitgliedstaaten nicht ausreichend erreicht werden können und dahcr wegen ihres Umfanges oder ihrer Wirkungen besser auf Gemeinschaftsebene erreicht werden können ....

Art. 3b EGV setzt mit den Kriterien "nicht ausreichend ", "besser " und »erforderlich" den geeigneten rechtlichen Rahmen, um das oben beschriebene Modell der Kompetenzausübung zu verwirklichen. Seine Interpretation in diesem Sinne vermag das Spannungsverhältnis zu lösen, das zwischen der Notwendigkeit einer umfassenden Kompetenz der EG für einen europaweiten grenzüberschreitenden Umweltschutz im Interesse des "Ökosystems EG * einerseits und der Gefahr zentralistischer Regelungen mit Effizienz- und Demokratiedefiziten andererseits besteht. Die Begrenzung der gemeinschaftlichen Maßnahmen durch das Kriterium der »Erforderlichkeit" stellt insofern sicher, daß im Rahmen des gemeinschaftlichen Umweltschutzes genügend Handlungsspiclraum für notwendige nationale oder regionale Differenzierungen mit dem Ziel einer örtlichen Schutzverstärkung bleibt.

95 Vgl. Ritter, a. a. O., S. 646; Salzwedel, NVwZ 1987, S. 276 (278)

96 Vel. dazu nur Epiney/Furrer, LuR 1992, S. 369 (400 ff.) mit umfassenden weiteren Nachweisen.

97 Hierzu z. B. Knemeycr, DVBI 1990, S. 449; Hcillzen, JZ 1991, S. 317.

98 Zur Auslcgung vgl. Sclimidluber/Hitzler, NVwZ 1992, S. 720 ff., sowıe speziell zur Umweltpolitik, Kahl, AuR 1993. S. 414 (436 ff.). 
Schließlich erscheint es notwendig, die Staatszielbestimmung um eine Formulierung zu ergänzen, daß die Natur »um ihrer selbst willen « zu schützen ist. Eine hierauf basierende Anerkennung von Eigenrechten der Natur ${ }^{99}$ könnte die Umwelt aus ihrer Abhängigkeit von mbetroffenen « Menschen und ihrer damit verbundenen Schutzlosigkeit, man crinnerc sich nur an die ergebnislose "Robbenklagc" im Jahre $1988^{100}$, erlösen. Überdies könnten die Eigenrechte das Vollzugsdefizit im Umweltrecht beheben helfen, indem sie die Klagemöglichkeiten erweitern ${ }^{101}$. Abgesehen davon gewinnt eine zum Umweltschut\% verpflichtete "Staatszielbestimmung " erst dadurch mehr als eine nur deklaratorische Bedeutung, daß sie sich von ihrer Menschenbczogenheit befreit und damit zur ökologischen "Staatszielbestimmung " wird. In ihr allein repräsentiert sich ein schrittweiser zu verwirklichender ethischer Anspruch, der Politik und Recht dazu zwingt, sich mit den Bedingungen und Grenzen eines Mitweltschutzes zu befassen. ${ }^{102}$ Unweltpolitik und Umweltrecht brauchen cinen "ethischen Unterbau«. Das Recht als Grundmuster der Politikgestaltung kann begrenzt neue ethische Grundlagen für das Verhalten der Menschen gegenüber der Umwelt in die Gesellschaft vermitteln und damit durch beständige Interaktionen kollektive Lernprozesse einleiten. ${ }^{103}$

Die Statuicrung von Eigenrechten ohne entsprechende Vertretungsbefugnisse wäre cine unvollkommene Regelung, da es zum Wesen des Rechts gehört, daß es gegenüber kollidierenden Intcressen geltend gemacht werden kann. Über Menschen, organisiert in Umweltverbänden, die die Rolle von Treuhändern und gesetzlichen Vertretern im Hinblick auf die "Eigenrechte" der Natur wahrnehmen ${ }^{104}$, könnte ein Rechtsschut $\iota$ der Umweltbelange erfolgen. Der Mensch als "trustee" mit der Funktion des "stewardship " betraut, ist eine Formulierung, dic auch im oben erwähnten "Environmental Imperative" des Europäischen Rates von Dublin 1990 vorkommt. Um Mißbräuche zu verhindern, setzt cine Vertretung durch Verbände staatliche Kontrollen und Anerkennung nach dem Vorbild des $\$ 29$ II BNatSchG voraus (Kriterien: demokratisch legitimierte Selbstorganisation, ausreichende Kompetenz und Kapazität) ${ }^{105}$. Vor diesem Hintergrund wird die Verankerung eines Verbandsklagerechts $^{106}$ in Art. I73 EGV für Verfahren vor dem EuGH, aber auch vor den Gerichten aller Mitgliedstaaten, unabdingbar ${ }^{107}$. An einem entsprechenden Richtlinien-Vorschlag arbeiter die EG-Kommission bereits ${ }^{10 x}$.

Die Umweltverbände als Vertreter der Belange nichtmenschlicher Naturgüter haben gegenüber einem etwa vom EP gewählten Unweltbeauftragten den Vorteil, daß sie gänzlich unabhängig von wechselnden parlamentarischen Mchrheiten und deren gelegentlich sachfremden Personalkompromissen agieren können ${ }^{109}$. Überdies ist vor Ort die Detailkenntnis von Problemen, Bedürfnissen und ökologischen Besonder-

99 Hierzu von Lersner, NVwZ 1988, S. 988 ; Christopher D. Stone, Umwelt vor Gericht, 1987; Bosselmann, Im Namen der Natur. Der Weg zum okologischen Rechtsstaat, 1992; sowie neuerdıngs Pernthaler, in: ders. NWeber/Wimmer, Umweltpolitik durch Recht, 1992, S. I (8 ff.).

100 Vgl. VG Hamburg, NVwZ 1988, S. 1058

101 Weber, IUR 1991, S. 81 (86).

102 V. Lersner, UTR Jahrbuch Bd. 12, 1990, S. ss (66).

103 In Anlehnung an Ritter, a. a. O., S. $648 \mathrm{f}$.

${ }_{104}$ Hierzu v. Lersner, UTR Jahrbuch Bd. 12, 1990, S. 55 (64 f.); Stone (Fn. 99), S. 38 ff.

tos Weber, IUR 1991, S.81 (86).

106 Vgl. dazu neuerdings ausfuhrlıch R. Wolf, ZUR 1994; S. I ff. m. w. N., sowie im internationalen Vergleich Winkelmann, ZUR 1994, S. $12 \mathrm{ff}$.

107 Vgl. v. Lersner, UTR Bd, 12,1990, S. 55 (64f.).

108 Vgl. 8. Bericht uber die Anwendung des Gemeinschaftsrechts, Anlage C zum Umweltschutz, KOM (91) 321 endg. Nr.99.

rog Weber, a. a. O.; v. Lersncr, a. a. O. 
heiten am größten. Entsprechend verhält es sich mit den Bereiligungs- und Mobilisierungschancen von Betroffenen und Bürgerinitiativen'ro. Auf der dezentralen Handlungsebene existiert die auf EG-Ebene vielfach vermißte Öffentlichkeit, die in der heutigen hochkomplexen Welt einen unabdingbaren Beitrag zur Qualität von Informationen, den darauf basiercnden Entscheidungen und damit zur Gouvcrnanz leisten kann und muß"'.

\section{Recht auf freien Zugang zu Umweltinformationen}

In engem Zusammenhang mit dem Thema Eigenrechte/Verbandsklage steht das Recht auf freien Zugang zu Unweltinformationen, das unabdingbare Voraussetzung für eine effektive Vertretung der Umweltinteressen ist. Ganz in diesem Sinne ging insbesondere das 4. Aktionsprogramm ${ }^{1 / 2}$ davon aus, durch eine Stärkung der Informations- und Beteiligungsrechte die Entwicklung notwendiger Umweltschutzmaßnahmen zu fördern sowie ihre Kontrolle und Akzeptanz zu verbessern. Entsprechend will die EG-Richtlinie über den freien Zugang zu Umweltinformationen"s nach der 4. Begründungserwägung ihrer Präambel »den Úmweltschuř verbessern «. Durch das von ihr gewährtc Zugangsrecht will die Richtlinic dic Transparenz des Umweltverwaltungshandelns erhöhen und die Informationsgrundlage der interessierten Öffentlichkeit verbreitern. Jedoch begründet die Informationsrichtlinie nur gegenüber den Behörden der Mitgliedstaaten einen entsprechenden Anspruch, die EG-Institutionen bleiben ausgespart. Angesichts der Tatsache, daß der Zugang zu Umweltinformationen der EG-Kommission nicht einfach ist, ist es begrüßenswert, daß dicse bei Vorlage des Vorschlags für die Informationsrichtlinie erklärte, sie werde eine entsprechende Gemeinschaftsregelung erlassen ${ }^{154}$. In der »Erklärung von Birmingham« vom Oktober 1992 hat auch der Europäische Rat unter dem Aspekt größerer Bürgernähe der EG die Notwendigkeit betont, die Arbeit der Gemeinschaftsorgane transparenter zu gestalten. Bei seiner Zusammenkunft in Edinburgh im Dezember 1992 ersuchte der Europäische Rat die Kommission nochmals, "Anfang nächsten Jahrcs ihre Arbeiten aufgrund der im Maastrichter Vertrag enthaltenen Erklärung über einen verbesserten Zugang zu den ihr und den anderen Gemeinschaftsorganen vorliegenden Informationen abzuschließen. "'"s Nach langen Vorarbeiten hat die Kommission nunmehr mit Beschluß vom 8. 2. 1994 einen Vcrhaltenskodex über den Zugang der Öffentlichkeit zu den der Kommission vorliegenden Dokumenten angenommen, der sich im wesentlichen an der Informations-Richtlinie orientiert. $^{116}$

ro Tritin, in: Calliess/Wegener (Hrsg.), Europasches Umwelterche als (hance, Taunusstein 1992; Weidenfeld, Europäische Defizite, europaische Perpektiven, 1988, S. 121; Liserbauer, in: Regionalisınus, 1978, S. 53 .

11 Club of Romc-Bericht 1991 (Fn. I), S. 104; allgemein hicrzu: Beck, Rusikogesellschaft, 1986, tnsbesondere $S .19,300$.

112 Abl. C 70 vom $18.3 \cdot 1987$, S. 3 ff.

I13 90/313/EWG, Abl. L is8 vom 23.6. 1990, S. 56 lf, dazu: Engel, NVwZ 1992, S. 1 i ff.; zur Limsetzung ins deutsche Recht: Wegener, IUR 1992, S. 21 Iff.

114 KOM (88) endg. vom 28.11, 1988, Nr. Ib.

11) Schlußfolgerungen des Vorsitzes-Birmingham, den 16.10.1992, S. 4, Anlage I, Nr. 3; Schlußfolgerungen des Vorsitzes-Edinburgh, 12. Dezember 1992, S. 42, Einleitung Nr. 7.

${ }_{13} 6$ Vgl. ABI EG Nr. L 46 v. 18. 2. 1994, S. 46 ; dazu Roller, elni-Report I/94, ZUR 1994, S.1. 
Durch "Öko-Steuern und Abgaben "18 sollen umweltgefährdende Produkte oder Herstellungsverfahren verteucrt werden, um das betriebswirtschaftliche Kalkül der Firmen und die Konsumgewohnheiten der Verbraucher entsprechend zu ändern. Was die größten Schäden verursacht oder die höchsten Risiken enthält, sollte am höchsten besteuert werden. Nicht mehr der Mehrwert, sondern der "Minderwcrt «im Sinne einer Minderung des Wertes unserer Umwclt durch den Verbrauch natürlicher Ressourcen - sollte besteuert werden"19.

Im Zuge des Binnenmarktes müssen die indirekten Steuern, die Mehrwertsteuer und die diversen Verbrauchssteuern harmonisiert werden. Auf diese Weise schrumpft der Spielraum der Mitgliedstaaten, "Öko-Steuern " cinzuführen" ${ }^{120}$. Ferner hindert die Scheu, Wettbewerbsnachteile für dic eigene Industrie zu begründen, so manchen Mitgliedstaat, das Mittel »Öko-Steuern « in der Umweltpolitik effizient einzusetzen. Umgekehrt hätten verschiedene nationale ${ }^{121}$ Vorhaben zur Einführung lenkender Energie- und Emissionsabgaben wettbewerbsverzerrende Auswirkungen auf den Binnenmarkt, so daß die Kommission nicht zuletzt aus dicsem Grund Handlungsbedarf auf Gemeinschaftsebene sah. Insbesondere in dem schon erwähnten s. Aktionsprogramm setzt die Kommission neben dem Ordnungsrecht aber auch verstärkt auf ökonomische Instrumente, wozu die Anwendung von ökonomischen und steuerlichen Anreizen gezählt wird ${ }^{12 z}$.

Im Juni r99r hatte die Fraktion der Grünen im EP einen Plan für einc europaweite Energiesteucr präsentiert. Diesc erste Gemeinschaftssteuer soll die Energieträger Kohle, Öl, Gas und Strom belasten. Die Steuer soll aus zwei Komponenten bestehen:

- einer allgemeinen Energiesteuer von r so DM je Tonne Rohöläquivalent

- einen Zuschlag von roo DM je Tonne Kohlenstoffgehalt, um die Bildung des klimaschädlichen $\mathrm{CO}_{2}$ durch finanzielle Lasten zu vermindern.

Die Energiesteuer soll stufenweisc eingeführt werden, um die Zusatzbelastung für die Verbraucher erträglich zu halten.

Einen weiteren Schritt in der Praxis unternahm die Kommission mit ihrem Vorschlag einer kombinicrten Energie/Kohlendioxid-Stcucr ${ }^{23}$ in ihrer Mitteilung an den Rat über "Eine Gemeinschaftsstrategie für weniger Kohlendioxidemissionen und mehr Energieeffizienz «"24. Soweit die EG die Iinführung einheitlicher Abgaben in den Mitgliedstaaten vorschreiben sollte, kann sie die Kompetenz für eine solche Steuer aus Art. rooa, 1 zos oder 99 EGVableiten. Sollen die Umweltsteuern jedoch als eigene Einnahmen der EG erhober werden, so fehlt dieser eine entsprechende Kompetenz, die mithin zu schaffen wäre'2s.

Derzeit wird im Rat über den Vorschlag der Kommission für einc Richtlinie zur Einführung einer Steuer auf Kohlendioxidemissionen und Energie ${ }^{126}$, die auf die

Ir 7 Vgl. hierzu allgemein den Überblick von Köck, NuR 1992, S. 412 m.w. N.

118 Vgl. hierzu auch Kloepfer, JZ 1991, S. 737; Kock; NuR 1992, S. 412.

$119 \mathrm{Zu}$ Vorschlagen fur weitere Kriterien und Ansatzpunkte bei der Ausgestaltung vgl. Europasches Umwcltbüro (EEB), Weißbuch der europaschen Umwelrverbande, EG-Bınncnmarkt und Umwelt, Brussel, Marz 1991, S. 13.

1 20 Vgl. Grabitz, in: Breuer (Hrsg.), UTR 7 (1989), S. 85.

121 Plane in Dánemark und der Bundesrepublik, s. dazu Breuer, DVB| 1992, S. $4^{8}$ s (496).

$122 \mathrm{KOM}(92)$ endg. VOL. If vom 3.4.1992, S. $72 \mathrm{f}$.

123 Ausführlich hierzu Pernice, RdE 1993, S. 45 ff.

124 SEK (9r) $1744 \mathrm{endg}$. vom 14. 10. 199 r.

125 Ausfuhrlich hierzu Hilf, NVwZ 1992, S. 105 ff.; Randzio-Plath, IUR 1991, S. 183 (185); Pernice, RdE r 993, S. $45 \mathrm{ff}$.

$126 \operatorname{KOM}(92) 226$ endgl. vom 30.6. 1992. 
Art. 99 und r 30 EWGV gestürzt ist, beraten. Da die Steuer zu Gunsten der Mitgliedstaaten erhoben werden soll, wäre es notwendig sicherzustellen, daß auf Gemeinschaftsebenc einheitliche Rahmenbedingungen für die Verwendung der Einnahmen aus der Steuer festgclegt werden. Neben dicser Frage sind die möglichen EG-internen und externen Wettbewerbsnachteile ein Streitpunkt, so daß eine konsequente Verwässerung des viclversprechenden Ansatzes zu befürchten ist, der mit den Vorstellungen des obigen Vorschlags der Grünen in EP, aber auch mit der EntschlieBung des EP vom 13. Juni 1991 nur noch wenig zu tun hat. ${ }^{127}$

Weitere Ansätze für Unwweltstcuern auf EG-Ebene könnten - worauf etwa die europäischen Umwcltverbände hinweisen ${ }^{128}$ - in der Umlage der Kfz-Steuer auf den Benzinpreis, einer Schwerverkehrsabgabe für den Güterkraftverkchr, einer Müllabgabe sowie einer Chlorabgabe (FCKW) bestehen. Das Aufkommen aus diesen Umweltabgaben und -steuern könnte dazu verwandt werden, Investitionen in umweltverträgliche Substitute in den entsprechenden Bereichen zu unterstützen. Ein Tcil der Einnahmen könnte in Hinblick auf die Ergcbnisse der Rio-Konferenz über Umwelt und Entwicklung vom Juni 1992 in einen globalen Umwelt- und Klimaschutzfonds eingezahlt werden.

Ein weitcres ökonomisch wirkendes Instrument der Umweltpolitik liegt in der $\mathrm{Ho}$ norierung ökologisch orientierter Produktion. Wer in einem umweltverträglichen Produktionsverfahren ökologiegerechte Produkte herstellt, muß in einer ökologischen Marktwirtschaft in die Lage versetzt werden, den hierin potentiell liegenden Konkurrenzvorteil auszunutzen. Er muß sein "mehr" an Umweltschutz den Verbrauchern vermitteln können, um dessen Lintscheidung insofern beeinflussen zu können. Sowohl im Interesse des Unternchmers als auch des Verbrauchers liegt es, eine Kennzeichnung aufgrund verläßlicher und klarer Kriterien der Unweltverträglichkeit im Wege "gesellschaftlicher Konsensfindung und Kontrolle festzulegen. Stichwörter sind insofern "Vertrauen und Dialog «. Die EG hat mit ihrer Verordnung betreffend ein gemeinschaftliches System zur Vergabe eines Umweltzeichens, die "Europäische Blume "'29, und mit dem Vorschlag der Kommission für eine Verordnung über die freiwillige Teilnahme gewerblicher Unternehmen an einer gemeinschaftlichen Umwelt-Betriebsprüfungsregelung (Öko-Audit) ${ }^{130}$ nunmehr erste Schritte in diese Richtung unternommen.

\section{6. Ökologie und Wettbewerbsrecht}

Im bereits erwähnten "Greening the Treaty «-Report der europäischen Umwcltverbände wurde vorgeschlagen, der Kommission die Kompetenz zu verleihen, staatliche Beibilfen (Subventionen) zu verbieten, wenn sie sich umweltschädigend auswirken (Neufassung der Art. 92-94 EWGV). Insofern würde es sich anbieten, Art. 92 I EWGV un die Formulierung zu ergänzen, daß eine Beihilfe, die niche umweltverträglich ist, mit dem Vertrag unvereinbar ist. Mindestens ebenso sinnvoll wäre es jedoch, einen klaren Ausnahmetatbestand in Art.92 II EWGV, der staatliche Beihilfen im Rahmen von umweltpolitischen Maßnahmen erlaubt, einzu-

\footnotetext{
127 Hierzu ausfuhrlich Randzio-Plath (Fn. 125), S. 183 f.

128 Ausfuhrlich Weißbuch der europaischen Umweltuerbande (Fn. I19), S. is f.

129 Verordnung (EWG) Nr. 880/92, ABIEG Nr. L 99 vom 11. 4. 1992, S. 1; ceilweise kritisch hierzu Roller, EuZW 1992, S. 499.

I 30 Ab EG Nr. L 168 vom 10. 7. 1993, S. 1; dazu mit ausgiebiger Analyse Fuhr, EuZW 1992, S. 468 (471 ff.); ders. EuZW 1993 , S. 859 .
} 
führen. Bisher ist hier die Rechtslage relativ kompliziert und undurchsichtig ${ }^{13}$.

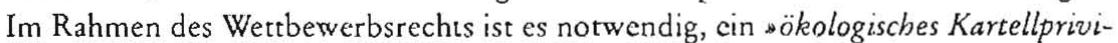
leg* einzuführen, da Umweltvcreinbarungen zwischen Unternehmen in der Regel einen wichtigen Zweck im öffentlichen Interesse erfüllen. Um hier derzeit verschlossene Freiräume für Unternehmen zu erschließen, wäre in Art.8s III EGV eine Erweiterung im Wertesystem der Freistellungsvoraussetzungen um eine ökologische Komponente vorzunehmen ${ }^{132}$.

Schließlich wäre es ökologisch wünschenswert, die Vergabe von finanziellen Mitteln aus den Strukturfonds, von der Europäischen Investitionsbank oder im Rahmen der EG-Forschungs- und Entwicklungspolitik einer nachprüfbaren UVP zu unterwerfen.

Alle diese Vorschläge sind letztlich bereits Ausfluß der erwähnten Querschnittsklausel'33. Jedoch empfiehlt sich in diesen rechtlich wie wirtschaftlich hochkomplizierten Bereichen einer konkretisierten Wirtschaftspolitik dic ausdrückliche Aufnahme einer ökologischen Komponente, um letztcre nicht als abstrakten Fremdkörper dem Vorwurf der Unvollzichbarkeit auszusetzen.

\section{Institutionelle Veränderungen}

a) Die Europäische Umveltagentur (EE.A)

Eine wichtige Entscheidung für den Bereich der europäischen Umweltpolitik wurde mit der F.G-Verordnung vom 7. Mai 1990'34 über dic Errichtung der EEA getroffen, die nunmehr ihre Arbeit mit Sitz in Kopenhagen aufgenommen hat ${ }^{135}$. Dicse europäische Umweltbehörde soll zunächst »nur « die Aufgabe haben, für die Organe der EG und für die Mitgliedstaaten Umweltdaten und andere umweltrelevante Informationen zu sammeln, aufzubereiten und zu analysieren. Nach den Vorstellungen von Umweltorganisationen und des EP soll die derzeitige Minimallösung jedoch ausgebaut und die EEA zu einer im EGV verankerten, möglichst unabhängigen Institution mit eigenen Exckutiv- und Kontrollfunktionen nach dem Vorbild der amerikanischen Bundesumweltbehörde: ${ }^{136}$ werden ${ }^{137}$.

Hierfür hat das EP den Grundstein gelegt, indem cs in der Verordnung durchsetzte, daß einheitliche Kriterien der Umweltbewertung eingeführt werden. Auch wenn die Mitgliedstaaten gegenwärtig nicht bereit sind, einc mit Exekutivbefugnissen ausgestattete EG-Umweltbehörde anzuerkennen, so gewährleisten einheitliche Bewertungskriterien für die nationalen Umweltbehörden doch einen Einfluß der EEA. Ferncr werden zwei EP-Parlamentarier im Führungsgremium der EEA sitzen und damit in bcschränktem Umfang eine demokratische Kontrolle gewährleisten. Schließlich wurde mit Art. 20 eine Revisionsklausel in die Verordnung eingefügt, die zwei Jahre nach ihrem Inkraftreten das Tor zum Ausbau der EEA-Kompetenzen öffnet und zu einer Entscheidung im obigen Sinne den Weg bereiten könnte.

131 Vgl, hierzu Grabitz, RIW 1989, S. 623.

132 In Anlehnung an Pernice, EuZW 1992, S. 139 (141).

133 Ebenso Kramer, Vortrag auf der Tagung "Unweltschurz. und andere Politiken " in Osnabruck am 26./27. 11. 1992; vgl. Tagungsbericht von Calliess/Wegener, ZUR 1993, S. 136 (1 37 ).

134 Verordnung (EWG) $1210 / 90$, Abl. EG vom 7. 5. 1990, L 120, S. I.

135 Ausfuhrlich zur Arbeit der Umweltagentur von Lersner, in: Calliess/Wegener (Hrsg.), Europaisches Umweltrecht als Chance, 1992, S. 81 if.

136 Dazu Petersen, KJ 1993 , S. 351.

137 Insofern zuruckhaltend von Lersner, in: Callıess/Wegener (Hrsg.) (Fn. 10), S. 87 ff. 
Unter dem Dach der EEA sollte mit Blick auf dic Rio-Folgckonferenzen eine "Agenda $2 \mathrm{I}$-Abteilung" eingerichtet werden, die der dringend erforderlichen Kooperation mit diesen Regionen durch ihre Expertise Rechnung tragen würde. Ferner könnten in allen Generaldirektionen der Kommission Fachabteilungen für Umweltfragen eingerichtet werden, die bereits in der Planungsphase von Maßnahmen deren Umweltverträglichkeit überprüfen und entsprechende Veränderungsvorschläge einbringen würden ${ }^{138}$.

Um den Vollzug des Gemeinschaftsrechts zu verbessern, gleichzeitig aber eine zentralistische "Superkontrollbehörde" zu vermeiden, sollte eine mit Kontroll- und Exekutivbefugnissen ausgestattete EEA von cinem Netzwerk nationaler Umweltinspektorate ergänzt werden ${ }^{i 39}$. Um den Umweltverbänden und interessierten Bürgern größtmöglichen Zugang zu den Umweltinformationssammlungen der EEA zu verschaffen, sollte eine der Informationsrichtlinic ähnliche Regelung für dic EEA geschaffen werden. Insofern könnte ein öffentlich zugängliches Kommunikationssystem eingerichtet werden.

\section{b) Ombudsman}

In Anlehnung an das dänische Modcll ${ }^{14^{\circ}}$ könnte das EP einen Ombudsman wählen, der als von der Kommission unabhängige Person den EP jährlich über den Vollzug des Umweltrechts in der EG zu berichten hätte. Der Ombudsman führt seinc Untersuchungen auf eigene Initiative, insbesondere aber auf Anregung von Bürgern und Umweltverbänden, durch, wobei er sich der Kapazitäten der EEA und der nationalen Umweltinspektoratc bedienen kann. Er könntc auch als Wahrer der Eigenrechte der Natur auftrcten. Seine Besonderheit liegt in der administrativen Unabhängigkeit, die auf der demokratischen Legitimation des EP basiert. Hiermit wäre auf der ökologischen Kontrollebene das rechtsstaatliche System der "checks and balances" (Gerichtshof, Parlament, Kommission und EEA) etabliert.

\section{c) Ökologischer Senat}

Um grundlegende Entschcidungen, ctwa übcr gesellschaftlich relevante unbckannte Großtcchnologien (z. B. Gentechnik) aus dem Dunkel von Bürokratie und Lobbyismus zu befreien, gleichzeitig aber auch um vom politischen Alltagsstreit losglöst gesellschaftlich tragfähige Konzepte und Strategien zur Lösung von Zukunftsproblemen zu cntwickeln, könnte ein Ökologischer Senat etabliert werden ${ }^{14}$. In ihn sollten Vertreter aus Wissenschaft und Technik sowie Vertreter aus Wirtschaft und Umweltverbänden je zur Hälfte von den nationalen Parlamenten und vom EP gewählt werden. Um ihre Unabhängigkeit von den Parteien und sonstigen Interessen zu gewährleisten, dürften sie keine aktiven Abgeordneten oder Vertreter eines Verbandes sein und würden einmalig auf zehn Jahre gewählt.

Die Aufgabe des Ökologischen Senats bestünde in Entschließungen zur Bewertung von Technologiefolgen, cinem Vetorecht bei Gisetzesvorhaben, das Rat und EP zu einer Neubefassung verpflichtet, sowie der Erstattung von Gutachten - aus cigener

138 Ebenso Weißbuch der europaischen Umweltverbande (Fn. 119), S. 8.

${ }_{3} 39$ Vgl. Macrory, Common Market Law Review, Vol. 29, No. 2, 1992, S. 347 (368)

140 Diese Anregung verdanke ich der danıschen Juristin Ditte Juul Jorgensen, EG-Kommission, Brussel; vgl. allgemein zum >Ombudsman, Schomerus, NuR 1989, S. 171.

141. Teilweise in Anlehnung an den Ókologischen Rat in Art. $53 \mathrm{~b}$ des Verfassungsentwurfs des Kuratorıums fur einen demokratisch verfaßten Bund deutscher Lander. Vonı Grundgeserz zur deutschen Verfassung, 1991, S. 45, 121, und das *Wissenschaftsparlament" bei Bohler, a. a. O., S. ross. 
Initiative oder auf Antrag des Rates bzw. eines Fünftels der Abgcordneten des EP -, in denen ökologische Konzepte und Strategien entwickelt werden. Um dieser Aufgabe gerecht werden zu können, müßte die Kommission umweltrelevante Vorschläge von grundlegender Bedeutung dem Ökologischen Scnat zu Beginn des gemeinschaftlichen Gesetzgebungsverfahrens zuleiten. Ein Veto des Ökologischen Senats müßte eine aufschiebende Wirkung für das gemeinschaftliche Gesetzgebungsverfahren in den Fällen zur Folge haben, in denen Rat oder Parlament sich über seine Einwände hinwegsetzen wollen. Wie ein solcher Ökologischer Senat in das fein ausdifferenzierte Gesetzgebungsverfahren der EG integriert werden könnte, muß unter Bcrücksichtigung des institutionellen Gleichgewichts anhand der dargestellten Grundsätze genau geprüft werden.

\section{Die Aktualität der ökologischen Rechtsgemeinschaft}

Dic Wende zur ökologischen Rechtsordnung unter den Stichworten "Umweltstaat ' $^{1 ; 2}$ und "ökologischer Rechtsstaat (143 $^{14}$ stellt sich nicht nur auf der Ebene des Nationalstaats, sondern auch für die EU/EG als große Herausforderung dar. Erste Ansätze auf EG-Ebene zu einem ökologischen Rechrsstaat sind zwar auszumachen, sie sind jedoch mit crheblichen Vollzugsdefiziten konfronticrt.

In Antwort auf die schleichende Umweltkatastrophe sind zwei einander ausschlieBende Entwicklungen denkbar: Die erste setzt auf den radikalen Umbau der Risikogesellschaft und auf eine Reform der Weltwirtschaft im Sinne eines "sustainable dcvelopment", mit Hilfe einer Rechtsordnung, die auf demokratischer und rechtsstaatlicher Grundlage konsequent ökologisch ausgerichtet ist. Die zweite besteht in der Gefahr cines Notstandsregimes in der Form einer "Ökodiktatur «: Unter autoritärem Frciheitsentzug zwingt ein nökologischer Leviarhan « den unwissenden Gesellschaftsmitgliedern von oben notwendige ökologische Anpassungen auf und bedient sich innen- wie außenpolitisch der Mittel der politischen und ökonomischen Unterdrückung, cinschließlich der Gefahr militärischer Konflikte ${ }^{1+4}$.

Um eine solche "Ökodiktatur"zu vermeiden und um einen demokratischen und sozialverträglichen Umbau der Risikogesellschaft zu ermöglichen, müssen die Instrumentarien des ökologischen Rechtsstaats jetzt etablicrt werden. Schon wenige Jahre später könnte diese Weichenstellung zum ökologischen Rechtsstaat zu spät kommen, so daß dann - in Reaktion auf die zu lange vernachlässigte schleichende Umweltkatastrophe - der Weg zu einer autoritären Ökodiktatur beschritten wird.

142 Ladenburger Kolleg "Umweltstaat «; dazu Kloepfer, DVBI 1994, S. 12; zum Schlußkongreỉ vgl. den Tagungsberiche von A. Lubbe, ZUR 1994, S. 42.

143 Tagung "Wege zum okologischen Rechtsstaat $\alpha$ des Institurs fur Umweltrecht, Birenten, 6./7. 2. 1993; dazu Tagungsbericht von Reiter, ZUR 1993, S. 190.

144 Vgl. Muller/Meyer-Abich, Kommt die Öko-Diktatur, in Die Zeı, Nr. $s$ vom 6. 4. 1990. 